\title{
Assistência ao Hematoma Subdural Crônico: Perfil de pacientes e diagnósticos de enfermagem segundo a NANDA-I
}

\author{
Chronic Subdural Hematoma Care: Patient profile and nursing diagnoses according to NANDA-I \\ Asistencia al hematoma subdural crónico: Perfil del paciente y diagnósticos de enfermería según la \\ NANDA-I
}

Recebido: 05/07/2021 | Revisado: 10/07/2021 | Aceito: 12/07/2021 | Publicado: 22/07/2021

\author{
João Cruz Neto \\ ORCID: https://orcid.org/0000-0002-0972-2988 \\ Universidade Regional do Cariri, Brasil \\ E-mail: enfjcncruz@gmail.com \\ Liana de Oliveira Barros \\ ORCID: https://orcid.org/0000-0001-9935-8122 \\ Universidade Estadual do Ceará, Brasil \\ E-mail: lianabarross@gmail.com \\ Sonia Samara Fonseca de Morais \\ ORCID: https://orcid.org/0000-0002-0146-4611 \\ Universidade Estadual do Ceará, Brasil \\ E-mail: enfsoniasamara@hotmail.com
}

\begin{abstract}
Resumo
Objetivo: O objetivo deste estudo foi identificar na literatura os diagnósticos e intervenções de enfermagem pelo perfil dos pacientes com HSDC. Método: Trata-se de uma revisão integrativa da literatura utilizando a estratégia PVO no período de junho a agosto de 2019 que teve como amostra seis estudos. As bases de dados consultadas foram: Literatura Latino-Americana e do Caribe em Ciências da Saúde, Medical Literature Analysis and Retrieval System Online, Índice Bibliográfico Español en Ciencias de la Salud e Banco de dados em Enfermagem. Resultados: Dentre as principais evidências relacionadas a doença estão os déficit neurológico e motor, cefaleias, delírios e crise convulsiva, com importantes complicações. O estudo reuniu doze diagnósticos de enfermagem e suas respectivas intervenções, quanto aos diagnósticos cinco deles foram relacionados ao domínio segurança e proteção. Nas intervenções, a reabilitação, controle hemodinâmico e da dor, cuidados com a saúde e prevenção de infecção foram as mais prevalentes. Conclusões: A equipe multiprofissional deve estar preparada para a assistência de maneira específica nos casos de hematoma subdural crônico, nesse sentido a sistematização da assistência de enfermagem tem papel essencial no direcionamento dos cuidados ao influenciar, essencialmente, na sobrevida do paciente.
\end{abstract}

Palavras-chave: Pacientes internados; Cuidados críticos; Hematoma subdural crônico.

\begin{abstract}
Objective: The objective of this study was to identify nursing diagnoses and interventions in the literature according to the profile of patients with HSDC. Method: This is an integrative literature review using the PVO strategy from June to August 2019, which had six studies as a sample. The databases consulted were: Latin American and Caribbean Literature in Health Sciences, Medical Literature Analysis and Retrieval System Online, Bibliographic Index Español en Ciencias de la Salud and Database in Nursing. Results: Among the main evidences related to the disease are neurological and motor deficits, headaches, delusions and seizures, with important complications. The study gathered twelve nursing diagnoses and their respective interventions, regarding the diagnoses five of them were related to the safety and protection domain. In interventions, rehabilitation, hemodynamic and pain control, health care and infection prevention were the most prevalent. Conclusions: The multiprofessional team must be prepared for assistance in a specific way in cases of chronic subdural hematoma, in this sense, the systematization of nursing care plays an essential role in directing care, essentially influencing the patient's survival.
\end{abstract}

Keywords: Inpatients; Critical care; Chronic subdural hematoma.

\section{Resumen}

Objetivo: El objetivo de este estudio fue identificar diagnósticos e intervenciones de enfermería en la literatura según el perfil de los pacientes con HSDC. Método: Se trata de una revisión integradora de la literatura utilizando la estrategia PVO de junio a agosto de 2019, que tuvo como muestra seis estudios. Las bases de datos consultadas fueron: Literatura Latinoamericana y Caribeña en Ciencias de la Salud, Sistema de Análisis y Recuperación de Literatura Médica en Línea, Índice Bibliográfico Español en Ciencias de la Salud y Base de Datos en Enfermería. Resultados: Entre las principales evidencias relacionadas con la enfermedad se encuentran déficits neurológicos y 
motores, dolores de cabeza, delirios y convulsiones, con importantes complicaciones. El estudio reunió doce diagnósticos de enfermería y sus respectivas intervenciones, con respecto a los diagnósticos cinco de ellos relacionados con el dominio de seguridad y protección. En las intervenciones, la rehabilitación, la hemodinámica y el control del dolor, la atención de la salud y la prevención de infecciones fueron las más prevalentes. Conclusiones: El equipo multiprofesional debe estar preparado para la asistencia de manera específica en casos de hematoma subdural crónico, en este sentido, la sistematización del cuidado de enfermería juega un papel fundamental en la dirección del cuidado, influyendo fundamentalmente en la supervivencia del paciente.

Palabras clave: Pacientes hospitalizados; Cuidado crítico; Hematoma subdural crónico.

\section{Introdução}

O hematoma subdural crônico (HSDC) é uma entidade patológica caracterizada por uma coleção encapsulada, com grau elevado de degeneração, e bem delimitada entre a dura-máter e a aracnoide (Farhat Neto et al., 2015). Esse problema de saúde é reconhecido pela Classificação Internacional das Doenças (CID), acometendo pessoas de diferentes idades e manifestando-se com variados graus de complexidade.

O fator tempo é essencial para a vida do paciente, uma vez que os estágios variam e o risco a vida se torna uma característica definida pelo pronto atendimento e o diagnóstico preventivo e diferencial (Alves et al., 2019). De acordo com o tempo que decorre entre o trauma e o diagnóstico de HSDC, os hematomas subdurais podem ser classificados em agudo (quando o diagnóstico é feito em até 72 horas após o evento traumático), subagudo (entre 72 horas e 20 dias) e crônico (mais de 20 dias após injúria (Farhat Neto et al., 2015;Pereira et al., 2015).

O HSDC tem como principal origem a lesão traumática das veias pontes em até $50 \%$ dos casos, queda sem trauma cranioencefálico direto e não traumático, sangramento originado de coagulopatia, tumores cerebrais, aneurismas intracranianos, malformações arteriovenosas e carcinomatose meníngea (C. Pereira et al., 2015). Nesse sentido, a assistência adequada e de forma individualizada são essenciais na intervenção precisa e na resolutividade dos eventos em pacientes com essa comorbidade.

Nesse contexto, faz-se necessário que o profissional de saúde seja capacitado e preparado para o acompanhamento, identificação e prevenção de agravos (Barbiani et al., 2016). O diagnóstico diferencial conta com o poder de entendimento da equipe sobre quais os cuidados ao paciente serão necessários, e com a ajuda de exames de imagem para abordagem precisa ao problema de saúde (Oliveira et al., 2016).

A enfermagem atua diretamente no cuidado pré e pós-cirúrgico prestado ao paciente, desde o levantamento de dados, o planejamento de metas para reabilitação, bem como a observância do líquido drenado e sua medição (Báo et al., 2019). Destaca-se ainda os cuidados com os curativos da ferida operatória e a promoção do conforto, seja na orientação da imagem corporal ou no esclarecimento de dúvidas, no ambiente hospitalar ou em domicílio (Oliveira et al., 2016).

Nos últimos anos é evidente o interesse de profissionais da saúde pela ampliação dos conhecimentos embasados por evidências científicas. A enfermagem é uma das áreas que mais se destacam no que se refere a produção científica, contendo um vasto acervo de publicações que podem contribuir de forma significativa na prática da assistência.

Apesar dos cuidados com hematomas subdurais estarem relacionados a práticas simples de média e baixa complexidade no ambiente hospitalar, existe uma lacuna no conhecimento sobre a assistência de enfermagem a esses pacientes, bem como a caracterização precisa dos entes relacionados a gestão do cuidado. Na literatura, são escassos os trabalhos relacionados a enfermagem e aos hematomas sendo este, portanto, o primeiro que faz esta correlação.

Estudos nessa perspectiva permitem a centralidade dos cuidados, elaboração do plano de metas, estratégias de intervenção e métodos de reavaliação que implicam diretamente na qualidade do serviço prestado e na promoção de conforto aos pacientes. Este estudo teve como objetivo foi identificar na literatura os diagnósticos e intervenções de enfermagem pelo perfil dos pacientes com HSDC. 


\section{Metodologia}

Realizou-se uma revisão bibliográfica integrativa em seis etapas: I) identificação do tema e seleção da hipótese ou questão de pesquisa para a elaboração da revisão integrativa; II) estabelecimento de critérios para inclusão e exclusão de estudos/amostragem ou busca na literatura; III) definição das informações a serem extraídas dos estudos selecionados/categorização dos estudos; IV) avaliação dos estudos incluídos na revisão integrativa; V) interpretação dos resultados; VI) apresentação da revisão/síntese do conhecimento (Mendes et al., 2019).

Nessa perspectiva, a questão norteadora que motivou esta revisão foi: Qual o perfil dos pacientes com HSDC submetidos a diagnósticos e intervenções de enfermagem? Estipulou-se a estratégia Population, Variables and Outcomes (PVO) para identificação da população, contexto e variáveis de interesse descritas no Quadro 1.

Quadro 1 - Descritores de assunto localizados no DeCS para os componentes da pergunta de pesquisa segunda a estratégia PVO. Brasil, 2021.

\begin{tabular}{|l|l|l|}
\hline Itens da estratégia & Componentes & DeCS \\
\hline Population & Pacientes & Pacientes Internados \\
\hline Variables & Assistência ao Paciente & Cuidados Criticos \\
\hline Outcomes & Cuidado ao HSDC & Hematoma subdural crônico \\
\hline
\end{tabular}

Fonte: Autores.

As bases eletrônicas de dados utilizadas neste estudo foram: Literatura Latino-Americana e do Caribe em Ciências da Saúde (LILACS), Medical Literature Analysis and Retrieval System Online (MEDLINE) via a National Library of Medicine National Institutes of Health (PUBMED), Índice Bibliográfico Español en Ciencias de la Salud (IBECS) e Banco de dados em Enfermagem (BDENF) durante o período de julho a agosto de 2019, utilizando o método de busca avançada e a categoria título, resumo e assunto.

Em cada base de dados, os descritores de assunto do Medical Subject Heading (MeSH) da PubMed e foram delimitados e cruzados, sendo utilizados: Inpatients, Critical Care e Hemantoma, Subdural chronic e seus DeCS, com os operadores booleanos AND e OR, de forma pareada por pesquisadores distintos.

A amostra foi composta por seis artigos que passaram por um processo de filtragem constituído pelos critérios de inclusão: artigos disponíveis eletronicamente com texto completo online; classificados como originais; publicados em português, inglês ou espanhol. Como critérios de exclusão cita-se os editoriais, as cartas ao editor e os estudos reflexivos, bem como estudos que não abordassem a temática investigada, sem limites temporais.

A discussão dos estudos foi feita seguindo os níveis de evidência (NE) pela proposição adaptada de Stillwell et al (2010): O NEI corresponde as metanálise e os ensaios clínicos controlados randomizados, NEII são os estudos clínicos nãorandomizados; NEIII são os estudos de coorte prospectivos e os estudos de caso-controle, NEIV série de casos e NEV opinião de especialistas, revisão narrativa e outros desenhos. Os dados foram descritos em tabelas e interpretados com base na literatura pertinente.

Utilizou-se o instrumento Preferred Reporting Items for Systematic Rewiew and Meta-Analyses (PRISMA) para demostrar o processo de busca e seleção dos estudos, conforme Figura 1. 
Figura 1 - Fluxograma do processo de busca e seleção dos artigos, Brasil,2021.
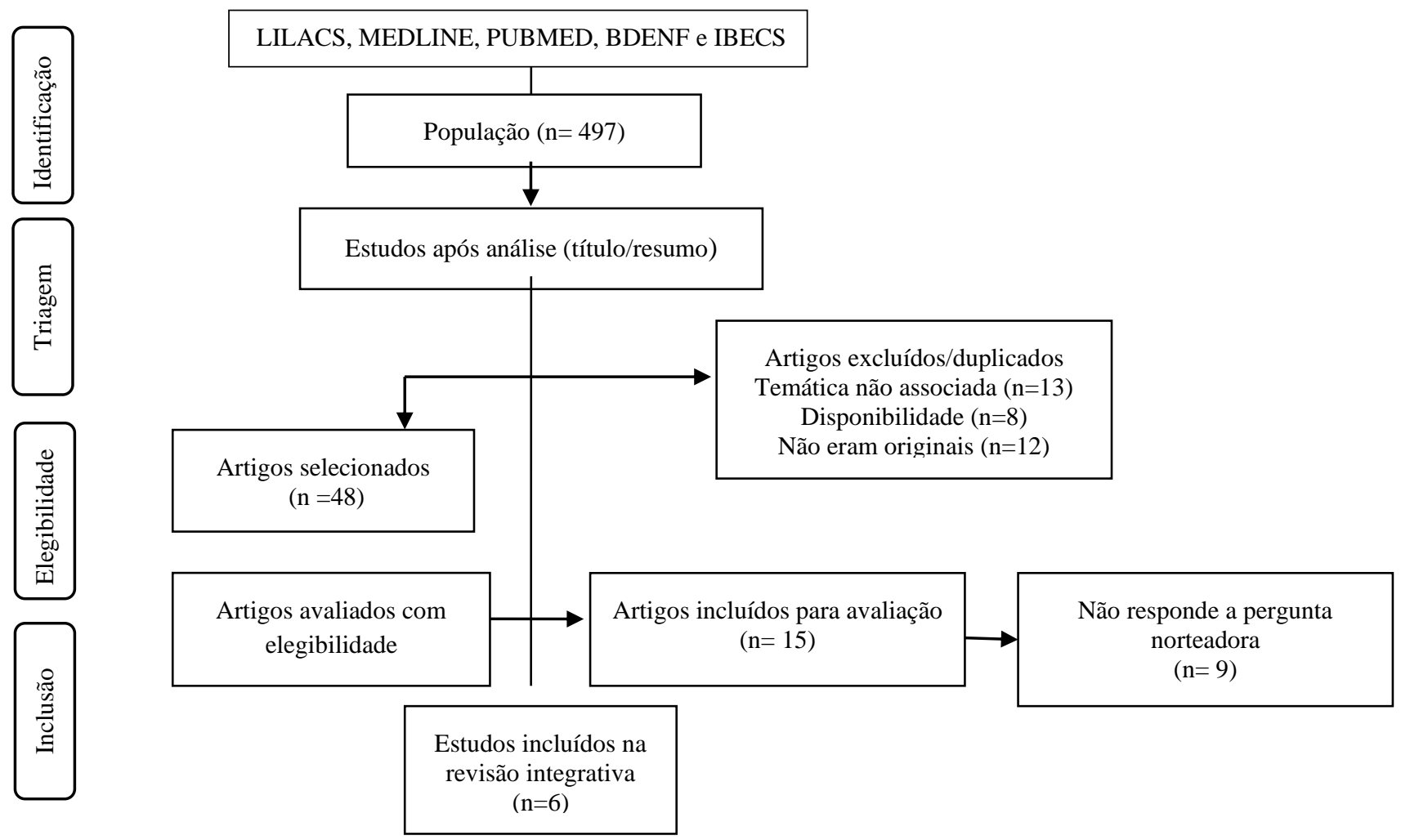

Fonte: Autores (2020).

Para sistematizar o processo de coleta de informações, utilizou-se um formulário próprio que identificou: título, autor, periódico, ano, nível de evidência (NE), objetivo, resultados e conclusões. A análise dos dados foi realizada criticamente buscando discutir os resultados dos diferentes estudos. Posteriormente, procedeu-se à síntese dos dados, apresentação dos resultados, interpretação e discussão de forma crítica e descritiva, fazendo associações entre os fatores contribuintes e não contribuintes para a assistência e cuidado ao paciente.

\section{Resultados}

A amostra foi constituída por 6 artigos, sendo composta por dois de 2018, dois de 2015, um de 2003 e um de 1990 distribuídos em 5 periódicos: Revista do colégio brasileiro de cirurgiões, Arquivos Brasileiros de Neurocirurgia, Jornal Brasileiro de Neurocirurgia, Arquivos de Neuro-Psiquiatria e Arquivos Médicos dos Hospitais e da Faculdade de Ciências Médicas da Santa Casa de São Paulo. (Quadro 2). 
Quadro 2 - Caracterização dos estudos incluídos, Brasil, 2021.

\begin{tabular}{|c|c|c|c|}
\hline $\begin{array}{l}\text { Título/Autores/ } \\
\text { Periódico/Ano/NE }\end{array}$ & Objetivo & Resultados & Conclusões \\
\hline $\begin{array}{l}\text { Hematoma subdural crônico: } \\
\text { análise epidemiológica e } \\
\text { prognóstica de } 176 \text { casos. } \\
\text { Farhat Neto et al. } \\
\text { Revista do Colégio Brasileiro de } \\
\text { Cirurgiões [online]. 2015/ III }\end{array}$ & $\begin{array}{lrr}\text { Caracterizar os pacientes } & \text { com } \\
\text { HSDC }^{b} & \text { submetidos a } \\
\text { Intervenção } & \text { Cirúrgica } \\
\text { Identificar os Indicadores } \\
\text { Prognósticos }\end{array}$ & $\begin{array}{l}\text { O HSDC foi causado por trauma } \\
\text { em } 52 \% \text { dos pacientes. Eram } \\
\text { hipertensos } 37,7 \% \text { dos pacientes e } \\
20 \% \text { possuíam alguma doença } \\
\text { neurológica. } 44 \% \text { apresentaram } \\
\text { cefaleia, alteração da consciência } \\
\text { ocorreu em } 40 \% \text { dos pacientes e } \\
\text { as alterações motoras, em } 27,5 \% \text {. }\end{array}$ & $\begin{array}{l}\text { A alteração de consciência foi a } \\
\text { alteração clínica mais comum } \\
\text { nos idosos e a cefaleia em não } \\
\text { idosos. A comorbidade mais } \\
\text { associada foi a HAS e a causa } \\
\text { mais frequente, o traumatismo } \\
\text { craniano. }\end{array}$ \\
\hline $\begin{array}{l}\text { Hematoma subdural crônico em } \\
\text { adultos jovens. } \\
\text { Pereira et al. } \\
\text { Arquivos Brasileiros de } \\
\text { Neurocirurgia. } 2015 \text { /III }\end{array}$ & $\begin{array}{l}\text { Analisar as particularidades do } \\
\text { HSDC em adultos jovens por } \\
\text { possuir uma apresentação } \\
\text { pouco frequente nessa faixa } \\
\text { etária. }\end{array}$ & $\begin{array}{l}\text { A cefaleia foi a queixa mais } \\
\text { comum (18 pacientes), seguida de } \\
\text { déficit neurológico focal (9), } \\
\text { alterações do nível de consciência } \\
\text { (6) e crises convulsivas (4) } \\
\text { Vinte e oito pacientes foram } \\
\text { tratados cirurgicamente e } 6 \\
\text { receberam tratamento } \\
\text { conservador. Vinte e sete } \\
\text { pacientes tiveram boa evolução, } 4 \\
\text { apresentaram déficit neurológico } \\
\text { focal, } 2 \text { apresentaram crise } \\
\text { convulsiva e houve um óbito. }\end{array}$ & $\begin{array}{l}\text { O hematoma subdural crônico é } \\
\text { frequente em adultos jovens. O } \\
\text { traumatismo cranioencefálico e } \\
\text { etilismo crônico foram as } \\
\text { principais causas de hematoma } \\
\text { subdural crônico em jovens. } \\
\text { Acomete mais o gênero } \\
\text { masculino. O tratamento de } \\
\text { escolha é cirúrgico. O } \\
\text { prognóstico é bom. }\end{array}$ \\
\hline $\begin{array}{l}\text { Manifestações Psiquiátricas do } \\
\text { Hematoma Subdural Crônico no } \\
\text { Idoso } \\
\text { Pereira } \text { et al } \\
\begin{array}{l}\text { Jornal Brasileiro } \\
\text { Neurocirurgia. 2018/V de }\end{array}\end{array}$ & $\begin{array}{l}\text { Identificar em uma revisão de } \\
\text { literatura, manifestações } \\
\text { psiquiátricas relacionadas ao } \\
\text { hematoma subdural crônico }\end{array}$ & $\begin{array}{l}\text { As manifestações psiquiátricas } \\
\text { em pacientes idosos com HSDC } \\
\text { são distúrbios cognitivos que } \\
\text { podem simular estado de delirium } \\
\text { ou de demência. } 88 \text { casos de } \\
\text { HSDC, onde } 40,58 \% \text { cursaram } \\
\text { inicialmente com quadro } \\
\text { psiquiátrico tipo confusão mental, } \\
\text { desorientação temporo-espacial, } \\
\text { amnésia e delírio onírico. }\end{array}$ & $\begin{array}{l}\text { O HSDC pode apresentar } \\
\text { manifestação exclusivamente } \\
\text { Psiquiátrica }\end{array}$ \\
\hline $\begin{array}{l}\text { Hematoma subdural crônico } \\
\text { Tratamento cirúrgico e } \\
\text { resultados em } 96 \text { pacientes } \\
\text { operados. } \\
\text { Salomão et al } \\
\begin{array}{l}\text { Arquivos de } \\
\text { Psiquiatria.1990/ IV }\end{array}\end{array}$ & $\begin{array}{l}\text { Verificar a evolução pós } \\
\text { cirúrgica em pacientes com } \\
\text { hematoma subdural crônico } \\
\text { submetidos a cirurgias. }\end{array}$ & $\begin{array}{l}78 \text { pacientes }(81,3 \%) \text { foram } \\
\text { considerados curados, } 6 \quad(6,2 \%) \\
\text { apresentaram sequelas e } 12 \\
(12,5 \%) \text { faleceram. Sequelas } \\
\text { neurológicas foram observadas } \\
\text { principalmente em pacientes } \\
\text { submetidos a reoperações }\end{array}$ & $\begin{array}{l}\text { Os autores chamam a atenção } \\
\text { para a ocorrência de hipotensão } \\
\text { intracraniana associada a } \\
\text { colapso cerebral. A importância } \\
\text { do diagnóstico precoce e } \\
\text { cirurgia imediata são } \\
\text { enfatizadas. }\end{array}$ \\
\hline $\begin{array}{l}\text { Hematoma subdural crônico } \\
\text { Estudo de } 161 \text { pacientes } \\
\text { operados e a relação com } \\
\text { alterações no coagulograma } \\
\text { Yasuda et al } \\
\text { Arquivos de Neuro-Psiquiatria. } \\
\text { 2003/ III }\end{array}$ & $\begin{array}{l}\text { Analisar a evolução de } \\
\text { pacientes com hematoma } \\
\text { subdural crônico em relação } \\
\text { aos achados do coagulograma. }\end{array}$ & $\begin{array}{l}\text { Pacientes com história prévia de } \\
\text { traumatismo cranioencefálico } \\
\text { apresentaram menor mortalidade } \\
\text { pós-operatória do que aqueles } \\
\text { sem tal antecedente. } \\
\text { Aproximadamente } 14,2 \% \text { deles } \\
\text { apresentavam antecedente de } \\
\text { etilismo. }\end{array}$ & $\begin{array}{l}\text { Os resultados ressaltam a } \\
\text { importância da avaliação pré- } \\
\text { operatória com o coagulograma } \\
\text { a fim de se corrigir possíveis } \\
\text { alterações. }\end{array}$ \\
\hline 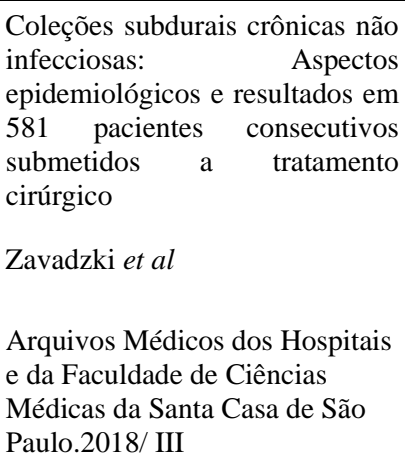 & $\begin{array}{l}\text { Traçar o perfil epidemiológico } \\
\text { dos pacientes diagnosticados } \\
\text { com coleções subdurais } \\
\text { crônicas, representadas por } \\
\text { HSDCr e HSD, submetidos a } \\
\text { tratamento neurocirúrgico no } \\
\text { nosso serviço, no período de } \\
2000 \text { a } 2014 \text {. }\end{array}$ & $\begin{array}{l}\text { Eram do sexo masculino } 74,18 \% \text {. } \\
\text { Em relação aos HSDC, a média } \\
\text { de idade foi } 61,6 \pm 21 \text { anos. A } \\
\text { trepanação foi o tipo de } \\
\text { intervenção mais utilizada }(95 \%) \text {, } \\
\text { seguido de craniectomia }(4,7 \%) \text {. }\end{array}$ & $\begin{array}{l}\text { Os HSDC representam a } \\
\text { maioria das coleções subdurais } \\
\text { submetidas a tratamento } \\
\text { cirúrgico. } \\
\text { Os idosos foram o grupo mais } \\
\text { acometido. O tratamento } \\
\text { cirúrgico de escolha foi a } \\
\text { trepanação. }\end{array}$ \\
\hline
\end{tabular}

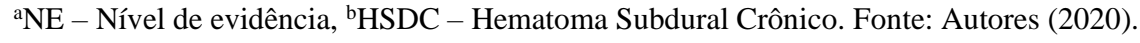


Em cinco estudos a principal causa do HSDC foi trauma cranioencefálico. Além disso, os estudos revelaram que outras possíveis causas envolvem o etilismo crônico, crise convulsiva, raquinestesia, acidentes automobilísticos e queda da própria altura, bem como em raros casos o uso de anticoagulantes orais.

Um estudo revelou o uso de medicações como da enzima conversora de angiotensina como possível fator associado ao HSDC. Os distúrbios mentais após a cirurgia do HSDC foram relatados em três estudos. Dois estudos revelaram que a Escala de coma de Glasgow variou de 3 a 14 durante o atendimento inicial até a alta hospitalar. Nos seis estudos, a prevalência do agravo se deu no sexo masculino.

A tomografia computadorizada é utilizada cinco estudos como método diagnóstico enquanto a ressonâncias magnéticas em apenas um estudo. $\mathrm{O}$ tratamento cirúrgico é indicado em quatro estudos com trepanação de um a três orifícios. Três estudos apontam que o HSDC ocorre bilateralmente.

Quanto a assistência de enfermagem, salienta-se diagnósticos e intervenções de enfermagem aplicáveis ao ambiente intra-hospitalar. Os diagnósticos de enfermagem estão descritos conforme a NANDA-I (2018-2020) e as intervenções pela NIC $6^{\mathrm{a}}$ ed., conforme apontado no Quadro 3.

Quadro 3 - Caracterização da assistência de enfermagem no HSDC.

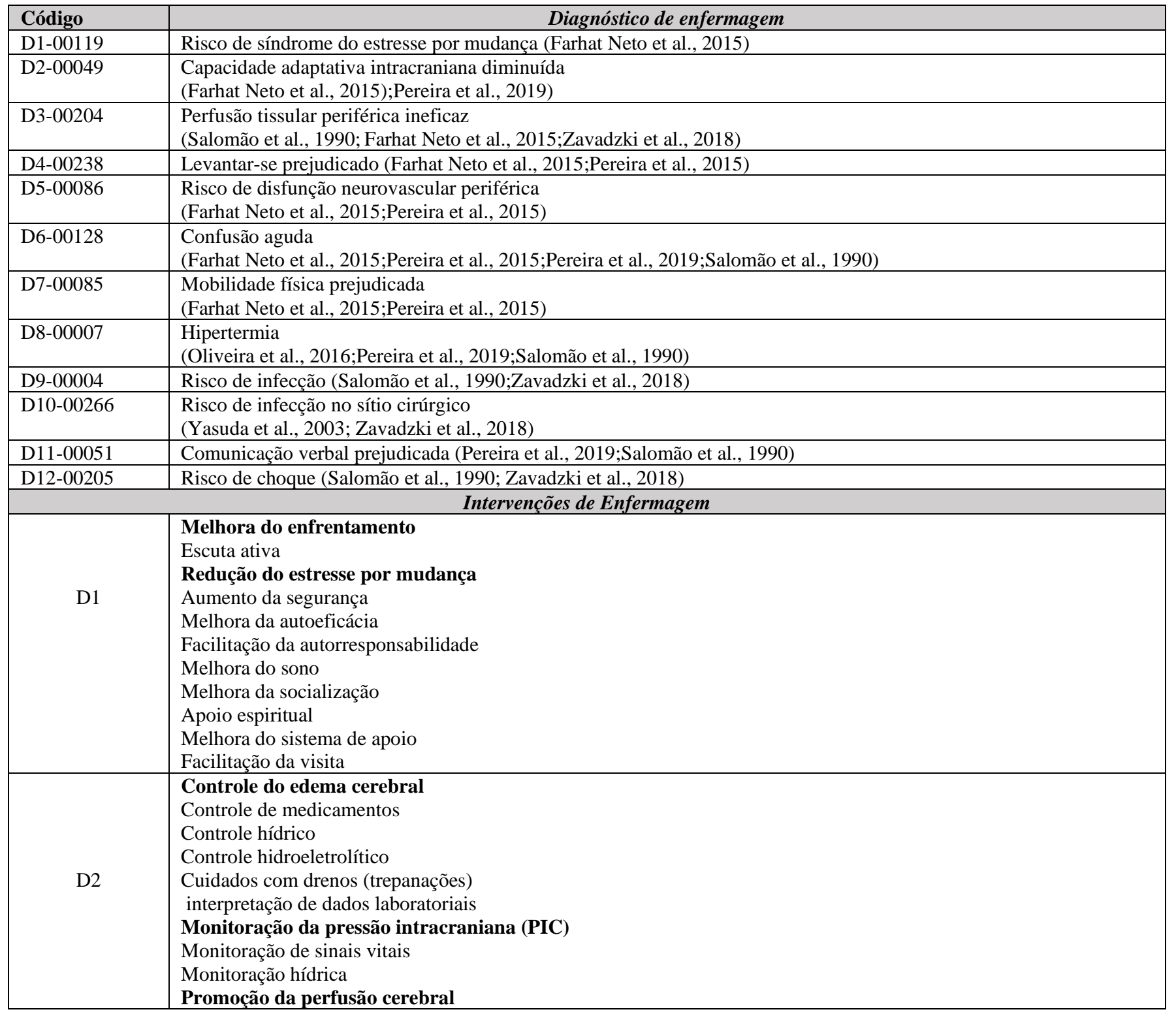




\begin{tabular}{|c|c|}
\hline & $\begin{array}{l}\text { Punção venosa } \\
\text { Supervisão } \\
\text { Terapia endovenosa (EV) } \\
\text { Adicionais } \\
\text { Controle de convulsões } \\
\text { Precauções contra convulsões controle acidobásico } \\
\text { Controle de vias aéreas } \\
\text { Redução da ansiedade } \\
\text { Proteção contra infecção }\end{array}$ \\
\hline D3 & $\begin{array}{l}\text { Controle da sensibilidade periférica } \\
\text { Controle da hipovolemia } \\
\text { Controle do choque } \\
\text { Controle hidroeletrolítico } \\
\text { Cuidados circulatórios: insuficiência venosa } \\
\text { Monitoração de sinais vitais } \\
\text { Monitoração neurológica } \\
\text { Posicionamento } \\
\text { Precauções circulatórias } \\
\text { Adicionais } \\
\text { Controle da dor } \\
\text { Controle de medicamentos } \\
\text { Administração de medicamentos } \\
\text { Punção venosa }\end{array}$ \\
\hline D4 & $\begin{array}{l}\text { Auxiliar o paciente a se levantar, deambular a uma distância específica e com o número específico de apoios } \\
\text { Estimular o paciente a se levantar-se espontaneamente quando adequado }\end{array}$ \\
\hline D5 & $\begin{array}{l}\text { Controle da sensibilidade periférica } \\
\text { Cuidados com a Tração/Imobilização } \\
\text { Monitoração neurológica }\end{array}$ \\
\hline D6 & $\begin{array}{l}\text { Abstinência de álcool } \\
\text { Administração de medicamentos } \\
\text { Contenção física } \\
\text { Controle da dor } \\
\text { Controle do delírio } \\
\text { Estimulação cognitiva } \\
\text { Melhora do sono } \\
\text { Monitoração de sinais vitais } \\
\text { Monitoração neurológica } \\
\text { Orientação para a realidade } \\
\text { Prevenção contra quedas }\end{array}$ \\
\hline D7 & $\begin{array}{l}\text { Assistência no autocuidado } \\
\text { Controle de energia } \\
\text { Controle do ambiente } \\
\text { Controle do humor } \\
\text { Promoção da mecânica corporal } \\
\text { Posicionamento } \\
\text { Controle da pressão } \\
\text { Promoção de exercício: alongamento }\end{array}$ \\
\hline D8 & $\begin{array}{l}\text { Controle de medicamentos } \\
\text { Controle do ambiente } \\
\text { Controle do choque } \\
\text { Controle de convulsões } \\
\text { Monitoração de sinais vitais } \\
\text { Prescrição de medicamentos } \\
\text { Proteção contra infecção } \\
\end{array}$ \\
\hline D9 & $\begin{array}{l}\text { Identificação de risco } \\
\text { Proteção contra infecção }\end{array}$ \\
\hline D10 & $\begin{array}{l}\text { Controle de infecção } \\
\text { Certificar-se de que o material é atual e atualizado } \\
\text { Certificar-se de que o material está esterilizado } \\
\text { Lavagem das mãos } \\
\text { Precauções padrão }\end{array}$ \\
\hline D11 & $\begin{array}{l}\text { Melhora da Comunicação: Déficit da Fala } \\
\text { Melhora da comunicação: déficit visual } \\
\text { Presença } \\
\text { Redução da ansiedade } \\
\text { Terapia de validação } \\
\text { Toque }\end{array}$ \\
\hline
\end{tabular}




\begin{tabular}{|l|l|}
\hline & Treinamento da memória \\
\hline & Controle de alergias \\
& Controle de infecção \\
& Monitoração de sinais vitais \\
Monitoração hídrica \\
Oxigenoterapia \\
& Precauçães contra sangramento \\
& Prevenção do choque \\
& Proteção contra infecção \\
& Redução do sangramento \\
& Redução do sangramento: ferimento \\
& Regulação hemodinâmica \\
& Reposição volêmica \\
& Supervisão \\
& Punção venosa \\
& Terapia endovenosa (EV) \\
\hline
\end{tabular}

Fonte: Autores (2020).

A taxonomia da NANDA-I comporta 13 domínios, 47 classes e 244 diagnósticos, destes a figura abaixo ilustra a distribuição dos diagnósticos citados neste estudo, Figura 2.

Figura 2 - Agrupamento de classes e domínios dos diagnósticos, 2021.

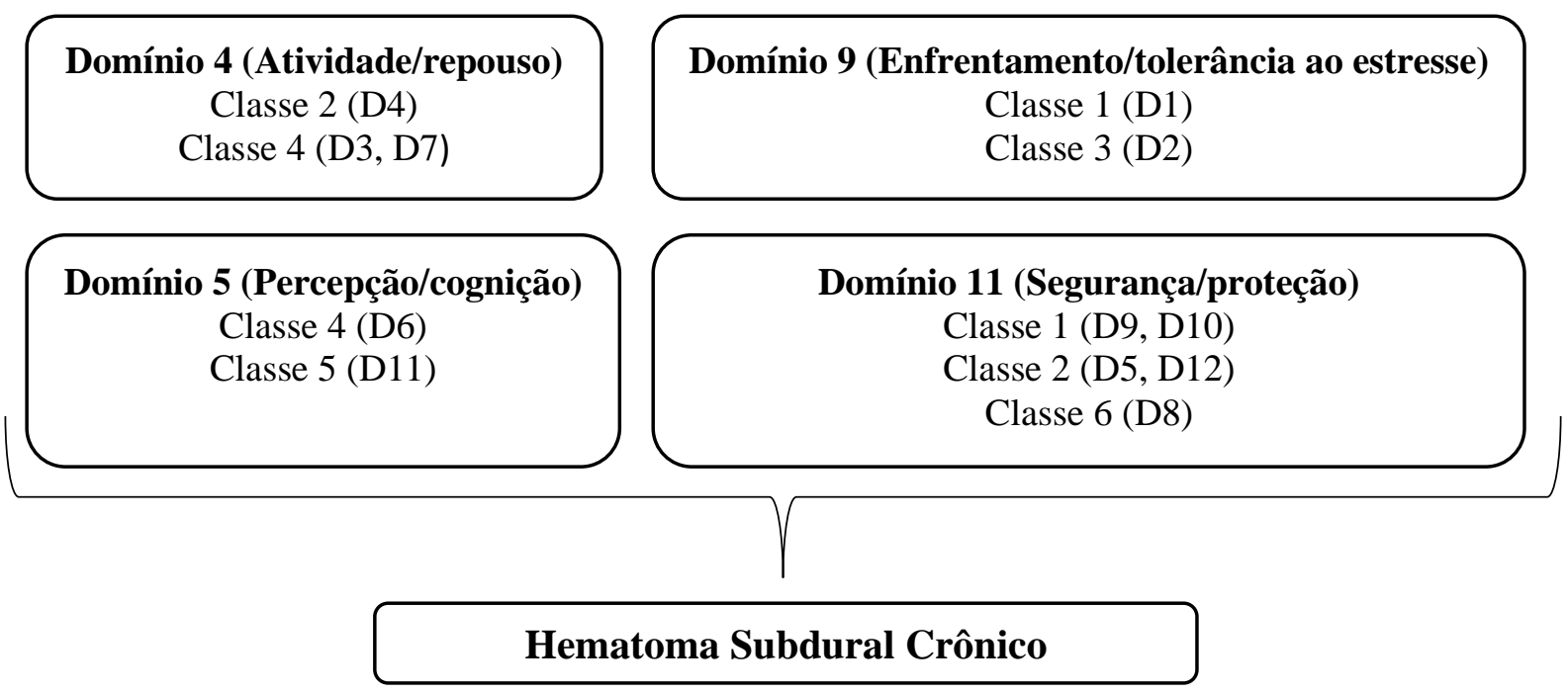

Fonte: Autores (2020).

Pela classificação, prevaleceu o domínio 11 com cinco diagnósticos. Destaca-se que o principal diagnóstico de enfermagem é a confusão aguda, seguido do risco de infecção devido procedimento cirúrgico e a perfusão tissular periférica ineficaz. O controle da dor, medicamentos e choque são intervenções apontadas para a maioria dos diagnósticos.

\section{Discussão}

Sempre que houver dificuldades na definição de um diagnóstico é importante considerar os aspectos do HSDC. Dentre estes, pode-se citar: História de traumatismo craniano ou movimentos bruscos, história de anticoagulação, distúrbios de antiagregação ou coagulação, congênita ou adquirida, alterações comportamentais inexplicáveis ou deterioração da consciência, convulsões recidivas e defeito motor progressivo e não proporcional (Castro-Rodríguez et al., 2016). 
A lesão traumática foi tratada com terapia anticoagulante ou antiplaquetária neles o principal sintoma foi o comprometimento cognitivo com localização no hemisfério esquerdo. O diagnóstico de um HSD pode ser fácil quando o trauma é recente e evidente, mas ele também pode passar despercebido e a anamnese adequada é essencial. Alguns sintomas são bem comuns como estado confuso agudo e disfasia global (Castro-Rodríguez et al., 2016).

O trauma cranioencefálico, constitui a primeira causa de complicações neurológicas no HSDC, as causas variam pelo uso de anticoagulantes orais, etilismo crônico, crises convulsivas e raquinestesia (Santana Silva \& Carvalho Filha, 2017). O que leva a necessidade de comprometimento do profissional tendo em vista os valores preditivos e escores de segurança do paciente, obtendo melhor taxa de sobrevida (Arruda et al., 2019;Sillero-Sillero \& Zabalegui, 2019).

Apesar de uma injúria considerada benigna, o risco de ser cronicamente progressiva dá destaque aos possíveis agravos que estão diretamente relacionados a outras patologias e a piora do quadro clínico (Lima et al., 2016). A cefaleia, hipoestesia, fraqueza, paresia, afasia e a alteração do nível de consciência são fatores recorrentes em pacientes neurocirúrgicos o que pode levar a paralisia dos nervos cranianos e convulsões (Herrero et al., 2017).

A média de tempo de permanência desse público é de, no mínimo, 25 horas com até 30 dias de internação hospitalar nos pós cirúrgicos (Herrero et al., 2017). De acordo com os estudos, as disfunções neurológicas atingem predominantemente homens na faixa de 43 anos (Sillero-Sillero \& Zabalegui, 2019).

$\mathrm{Na}$ população idosa o risco aumenta para a HSDC quando associada a atrofia cortical e aumento da fragilidade venosa, sendo assim, os indicadores estão relacionados a hemostasia sanguínea; a ruptura nas veias pontes com características de liquefeito até a sangue fresco (Zavadzki et al., 2018). O sangue também pode apresentar-se conforme a ação da fibrina, como formas hipodensa, isodenso ou hiperdenso (Farhat Neto et al., 2015).

A monitorização continua depende do prognóstico clínico do paciente. Nestes casos, o eletrocardiograma tem importante funcionalidade ao identificar alterações elétricas advindas do coração e consequentemente repercussões cerebrais pela demanda de sangue e oxigênio, como também outros exames complementares (Arruda et al., 2019).

A localização dos hematomas se dá principalmente de forma bilateral, podendo também apresentar-se de outras maneiras (Farhat Neto et al., 2015;Pereira et al., 2015). Esse dado está de acordo com os estudos encontrados nessa pesquisa. Os achados bilaterais ainda podem apresentar na linha média com prosseguimento para manobras cirúrgicas/conservadoras que indicam sedação com Fentanil ou Midazolam para diminuir a mortalidade (Arruda et al., 2019).

Dependendo da cirurgia a mortalidade pode variar de $1,6 \%$ a 20,5\%, nesses casos, observar a infecção de ferida, infecção urinária, lesão por pressão e a infecção respiratória se faz necessário para evitar complicações e morbimortalidade (Sillero-Sillero \& Zabalegui, 2019). O uso do sistema fechado de drenagem sem vácuo é um fator significante na prevenção de infecções e recidivas (Farhat Neto et al., 2015). Nesse sentido, a enfermagem perioperatória tem papel importante na segurança do paciente (Sillero-Sillero \& Zabalegui, 2019).

Algumas comorbidades também podem agravar os riscos da doença. Dentre as principais, observamos a hipertensão, a doença neurológica, vasculares e neoplasias que somam 77,7\% de agravantes (Farhat Neto et al., 2015). Cada manifestação do HSDC requer um cuidado individualizado e prestado com segurança e precisão, principalmente em pacientes que apresentam sinais de déficits neurológicos ou focais onde as práticas paliativas promovem maior qualidade de vida (Pereira et al., 2015).

Os conhecimentos acerca das respostas humanas são de competência do profissional de saúde, especialmente dos enfermeiros ao prestar assistência integral segura, seja por meio de protocolos ou da prática profissional (Caetano et al., 2020). Nos pacientes neurológicos os diagnósticos que mais se apresentam pertencem ao sono/repouso e segurança/proteção com foco no risco de infecção (Herrero et al., 2017).

Quando se realiza um trabalho consciente, com interesse de ouvir e compartilhando sentimentos os pacientes acatam os conselhos considerados uteis ao seu tratamento permitindo o melhor cuidado em saúde (Lima et al., 2016). Destaca-se que a 
temática constitui um problema de saúde pública e atinge pessoas em fase economicamente ativa causando gastos ao sistema público (Santana Silva \& Carvalho Filha, 2017; Bonelli et al., 2020).

O estudo teve por limitações a quantidade de estudos relacionados ao tema e a deficiência de publicações acerca da assistência de enfermagem ao agravo em estudo. Acrescenta-se que o conhecimento cientifico deve ser elucidado pelas melhores evidências na área e por isso a elaboração de estudos nesta perspectiva fortalece as ações e serviços em saúde ao fomentar a criação de indicadores pela prática clínica.

Aponta-se que a inclusão de estudos que realizem abordagens práticas do conhecimento à sistematização da assistência de enfermagem, fortalece a oferta dos cuidados e permite a adequação de prerrogativas que subsidiam a prática profissional, além de favorecer a construção de uma linguagem padronizada que vislumbre o raciocínio clinico e responda as necessidades humanas do processo saúde e doença.

\section{Conclusão}

A equipe multiprofissional deve estar preparada para a assistência de maneira específica e eficaz aos casos de HSDC e os fatores ligados a etiologia da doença tendo em vista o prognóstico clinico. Nesse sentido, o aprimoramento de medidas terapêuticas pode ser essencial na tomada de decisão e impacta diretamente na sobrevida do paciente.

Ressalta-se a investigação dos principais sinais e sintomas do problema com foco importante a cefaleia persistente que não cessa com analgésicos. Assim também, colabora-se aos achados a tomografia computadorizada como principal método diagnostico. Destaca-se os cuidados nos tempos três tempos cirúrgicos como uma tarefa multiprofissional, muitas vezes designada aos profissionais de enfermagem.

O estudo evidenciou que as alterações motoras e psicológicas estão presentes em grande parte dos acometidos pelo HSDC. Por isso, os diagnósticos e intervenções possuem foco na confusão aguda, estresse e mobilidade prejudicada, além de fatores ligados ao processo cirúrgico. As intervenções de enfermagem focam na reabilitação do paciente, monitorização hemodinâmica, prevenção de agravos e cuidados com a administração de medicamentos devido ao risco de infecção.

Sugere-se que novos estudos contemplem as atividades de enfermagem ligadas ao pré e pós-operatório, como também a abordagem da sistematização da assistência de enfermagem com a implementação de diagnósticos e intervenções no contexto multidisciplinar e com públicos de diferentes faixas etárias a fim de contribuir com a prática clínica e o desenvolvimento de instrumentos que favoreçam a prática profissional.

\section{Referências}

Alves, R. S. F., Cunha, E. C. N., Santos, G. C., \& Melo, M. O. (2019). Cuidados Paliativos: Alternativa para o Cuidado Essencial no Fim da Vida. Psicologia: Ciência e Profissão, 39, 1-15.

Arruda, P. L. de, Xavier, R. de O., Lira, G. G., Arruda, R. G. de, Melo, R. A. de, \& Fernandes, F. E. C. V. (2019). Evolução clínica e sobrevida de pacientes neurocríticos. Revista Da Escola de Enfermagem Da USP, 53, 1-8.

Báo, A. C. P., Amestoy, S. C., Moura, G. M. S. S. de, \& Trindade, L. de L. (2019). Quality indicators: tools for the management of best practices in Health. Revista Brasileira de Enfermagem, 72(2), 360-366.

Barbiani, R., Nora, C. R. D., \& Schaefer, R. (2016). Práticas do enfermeiro no contexto da atenção básica: Scoping review. Revista Latino-Americana de Enfermagem, 24.

Bonelli, M. A., Silva, I. C. C., Fernandes, T. H. T., Schwarz, T., \& Costa, F. S. (2020). Acute-on-chronic subdural cranial hematoma in a dog: Case report. Arquivo Brasileiro de Medicina Veterinaria e Zootecnia, 72(1), 131-135.

Caetano, J., Raimundo, L. J., Oliveira, R. J. T. de, Lessa, G., Nandi, K. Z., \& Santos, J. G. dos. (2020). Implementation of a safe surgery protocol: experience report. Revista de Enfermagem Da UFPI, 9(1), 98.

Castro-Rodríguez, C., Román-Pena, P., Arán-Echabe, E., \& Gelabert-González, M. (2016). Hematoma subdural crónico en pacientes muy ancianos. Revista Espanola de Geriatria y Gerontologia, 51(6), 309-316.

Farhat Neto, J., Araujo, J. L. V., Ferraz, V. R., Haddad, L., \& Veiga, J. C. E. (2015). Hematoma subdural crônico: Análise epidemiológica e prognóstica de 
Research, Society and Development, v. 10, n. 9, e13110917915, 2021

(CC BY 4.0) | ISSN 2525-3409 | DOI: http://dx.doi.org/10.33448/rsd-v10i9.17915

176 casos. Revista Do Colegio Brasileiro de Cirurgioes, 42(5), 283-287.

Herrero, S., Carrero, E., Valero, R., Rios, J., \& Fábregas, N. (2017). Postoperative surveillance in neurosurgical patients - usefulness of neurological assessment scores and bispectral index. Brazilian Journal of Anesthesiology, 67(2), 153-165.

Lima, A. C. M. A. C. C., Silva, A. L. da, Guerra, D. R., Barbosa, I. V., Bezerra, K. de C., \& Oriá, M. O. B. (2016). Diagnósticos de enfermagem em pacientes com acidente vascular cerebral: revisão integrativa. Revista Brasileira de Enfermagem, 69(4), 785-792.

Mendes, K. D. S., Silveira, R. C. de C. P., \& Galvão, C. M. (2019). Use of the bibliographic reference manager in the selection of primary studies in integrative reviews. Texto \& Contexto - Enfermagem, 28(e20170204), 1-13.

Oliveira, F. P. de, Oliveira, B. G. R. B. de, Santana, R. F., Silva, B. de P., \& Candido, J. de S. C. (2016). Classificações de intervenções e resultados de enfermagem em pacientes com feridas: mapeamento cruzado. Revista Gaúcha de Enfermagem, 37(2), 1-9.

Pereira, C., Santos, J., Santos, A., \& Passos, R. (2015). Hematoma subdural crônico em adultos jovens. Arquivos Brasileiros de Neurocirurgia: 34(01), 025029.

Pereira, C. U., Pereira, F. D. A., Rabelo, N. N., Rabelo, N. N., \& Oliveira, D. M. D. P. (2019). Manifestações Psiquiátricas do Hematoma Subdural Crônico no Idoso. Revisão da literatura. JBNC - jornal brasileiro de neurocirurgia, 29(01), 18-22.

Salomão, F., Leibinger, R., \& Lynch, J. (1990). Tratamento cirúrgico e resultados em 96 pacientes operados. Arq neuro-psiquiat. (48)1: 91-96.

Santana Silva, F., \& Soares Sousa Carvalho Filha, F. (2017). Trauma skull brain as a problem of public health: an integrative review of the literature. Rev. Reon Facema. 3(1), 389-395.

Sillero-Sillero, A., \& Zabalegui, A. (2019). Safety and satisfaction of patients with nurse's care in the perioperative. Revista Latino-Americana de Enfermagem, 27. https://doi.org/10.1590/1518-8345.2646.3142

Stillwell, S. B., Fineout-Overholt, E., Melnyk, B. M., \& Williamson, K. M. (2010). Evidence-Based Practice, Step by Step: Searching for the Evidence. AJN, American Journal of Nursing, 110(5), 41-47.

Yasuda, C. L., Morita, M. E., Nishimori, F. Y., Yasuda, A. M., \& Alves, H. L. (2003). Hematoma subdural crônico: estudo de 161 pacientes operados e a relação com alterações no coagulograma. Arquivos de Neuro-Psiquiatria, 61(4), 1011-1014.

Zavadzki, T. M., Costa, G. H. F. da, Rodrigues, L. H. D. S., Xander, P. A. W., Veiga, J. C. E., \& Aguiar, G. B. de. (2018). Coleções subdurais crônicas não infecciosas: Aspectos epidemiológicos e resultados em 581 pacientes consecutivos submetidos a tratamento cirúrgico. Arquivos Médicos Dos Hospitais e Da Faculdade de Ciências Médicas Da Santa Casa de São Paulo, 63(2), 85. 